\title{
MUDANÇAS EPISTEMOLÓGICAS NA ARQUITETURA: ENTRE ARQUIVOS, EXPOSIÇÕES E PUBLICAÇÕES
}

Epistemological changes in architecture: between archives, exhibitions, and publications

Cambios epistemológicos en la arquitectura: entre archivos, exposiciones y publicaciones

EDUARDO AUGUSTO COSTA ${ }^{\mathrm{l}^{*}}$

DOI: https://doi.org/10.1590/S2178-149420210107

Universidade de São Paulo - São Paulo (SP), Brasil.

*Professor doutor MS-3.1 do Departamento de História da Arquitetura e Estética do Projeto da Faculdade de Arquitetura e Urbanismo da Universidade de São Paulo, desenvolve pesquisa vinculada ao Programa Jovem Pesquisador da Fundação de Amparo à Pesquisa do Estado de São Paulo (eduardocosta@usp.br).

(D) https://orcid.org/0000-0002-7909-0496

Artigo recebido em 01 de setembro de 2020 e aprovado para publicação em 11 de novembro de 2020. 


\title{
RESUMo
}

Este artigo explora as mudanças epistemológicas da arquitetura ocorridas nas últimas cinco décadas. Com base na leitura de artigos científicos, textos críticos, políticas e práticas contemporâneas, identifica-se que houve expansão no entendimento do que vem a ser arquitetura e reconhece-se que a disciplina foi influenciada pelas mudanças ocorridas nas ciências humanas, com sensível atenção à cultura visual. Nesse sentido, destacam-se três domínios como expressões articuladas e estruturantes da prática contemporânea dos arquitetos, historiadores e críticos: os arquivos, as exposições e as publicações. Desse modo, busca-se destacar a centralidade da cultura visual nessa nova cultura arquitetônica.

PALAVRAS-CHAVE: Arquitetura; Cultura Visual; Historiografia; Arquivos; Exposições; Publicações.

\begin{abstract}
This paper explored epistemological changes in architecture over the past five decades. From scientific articles, critical texts, policies, and contemporary practices, it identifies that there has been an expansion in the understanding of what architecture is and recognizes that the discipline was influenced by the changes that occurred in the human sciences, with keen attention to visual culture. In this sense, the paper highlights three domains as articulated and structuring expressions of the contemporary practice of architects, historians, and critics: archives, exhibitions, and publications. In this way, it seeks to highlight the centrality of visual culture in this new architectural culture.
\end{abstract}

KEYWORDS: Architecture; Visual Culture; Historiography; Archives; Exhibitions; Publications.

\section{RESUMEN}

Este artículo explora los cambios epistemológicos en la arquitectura durante las últimas cinco décadas. A partir de artículos científicos, textos críticos, políticas y prácticas contemporáneas, identifica que ha habido una expansión en la comprensión de lo que es la arquitectura y reconoce que la disciplina fue influenciada por los cambios ocurridos en las ciencias humanas, con especial atención a la cultura visual. En este sentido, el trabajo destaca tres dominios articulados y estructurantes expresiones de la práctica contemporánea de arquitectos, historiadores y críticos: archivos, exposiciones y publicaciones. Busca resaltar la centralidad de la cultura visual en esta nueva cultura arquitectónica.

PALABRAS CLAVE: Arquitectura; Cultura Visual; Historiografía; Archivo; Exposiciones; Publicaciones. 


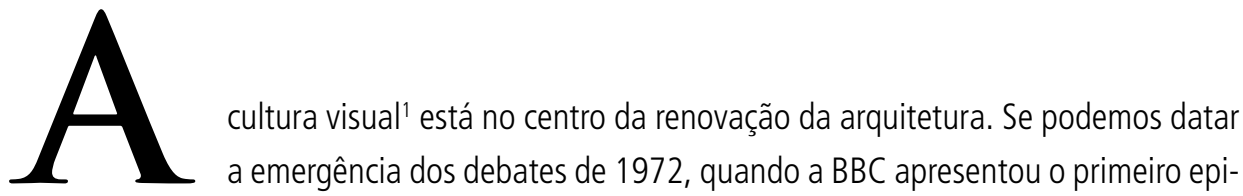
sódio da série Ways of Seeing, organizada por John Berger², arquitetos, críticos e historiadores da arquitetura, também naquele mesmo período, passaram a incorporar reflexões correlatas em suas produções e debates. É certo que a arquitetura, enquanto atividade prática, aplicada, manteve relação estreita com a visualidade ao longo de toda sua história. Da noção de beleza - venustas — referenciada como um dos três princípios da arquitetura clássica segundo o tratado de Vitrúvio, escrito no século I antes de Cristo³, aos galpões decorados descritos na clássica obra Learning from Las Vegas (Venturi, Scott Brown e Izenour, 1972), identifica-se que essa relação é duradoura e constitutiva da própria disciplina. Mas foi a partir da segunda metade do século XX que uma série de mudanças ocorreram no campo disciplinar, contribuindo para uma transformação na própria compreensão do que vem a ser arquitetura.

0 reconhecimento de tal mudança acompanha a produção das últimas décadas do século XX e vem ganhando força com a publicação de uma série de livros, números de revistas especializadas, a organização de seminários e eventos científicos, a formação de grupos e laboratórios de pesquisa, a curadoria de exposições, ações institucionais e culturais, além de estratégias empreendidas pelos próprios arquitetos e seus escritórios. Nestas duas primeiras décadas do século XXI, quando se revisita historicamente o que foram e como se delinearam as experiências radicais dos anos 1960-1970 e do pós-modernismo na arquitetura, historiadores vêm melhor circunscrevendo a centralidade da visualidade. É este o interesse primeiro, por exemplo, da historiadora Kester Rattenbury, que explicita seu ponto de vista já no título de seu livro This is not architecture. Numa referência à clássica obra de Magritte — La trahison des images, de 1929, conhecida pela frase estampada abaixo de um cachimbo, "c'est ne pas une pipe" - que suscitou uma série de debates nas artes (Bowman, 1985) e nas ciências humanas (Foucault, 1983), a historiadora ainda vai além ao apresentar seu argumento já no frontispício do livro:

A arquitetura é discutida, explicada e identificada inteiramente por meio de suas representações. De fato, essas representações são frequentemente tratadas como se fossem a própria arquitetura. Um enorme status é dado ao projeto imaginário, ao autêntico conjunto de fotografias ou à eminente avaliação crítica. Isso é um paradoxo. A arquitetura está fundamentalmente preocupada com a realidade física, mas discutimos e até definimos arquitetura (em oposição à construção) através de um elaborado construto de representações midiáticas: fotografia, jornalismo, crítica, exibição, história, livros, filmes, televisão e teoria crítica. (Rattenbury, 2002: i). 
O texto apresentado por Rattenbury evidencia a relação que a arquitetura passou a estabelecer com outras linguagens. Nesse paradoxo entre a materialidade e suas representações, os historiadores da arquitetura ampliaram o foco analítico, compreendendo que as leituras e interpretações dos conjuntos edificados, da matéria construída, passam também pelas mediações estabelecidas com um grande conjunto de representações. É essa também a leitura da historiadora Léa-Catherine Szacka, para quem "[...] a arquitetura opera através de um complexo de diferentes mídias - exposições, como também livros, revistas, filmes, televisão e novas mídias" (Szacka, 2016: 15).

Aqui, a questão ganha outros significados. Se para Rattenbury há o reconhecimento de que a arquitetura possui uma dimensão física inerente a ela e que, ainda que mediada por representações, é essa a preocupação fundamental dos arquitetos e dos historiadores, para Szacka a questão colocada tem outra natureza. A arquitetura, nesse caso, equaciona diversos artefatos e linguagens com base em um conjunto de agenciamentos. Essa dimensão é importante para as avaliações e leituras contemporâneas - de historiadores, críticos e também dos próprios arquitetos - , uma vez que não se debatem apenas objetos - imóveis - mas projetos idealizados que não chegam a ser concretizados. Ou seja: além da complexidade territorial imposta pelo corpus materializado, os historiadores debatem projetos que não foram construídos - projetos imaginários, como colocado por Rattenbury - , seja por suas dimensões utópicas, seja por circunstâncias político-culturais. No entanto, muitos desses projetos não realizados ganham relevância no campo por suas críticas, elaborações teóricas, publicações, desenhos, exposições e todo um conjunto de manifestações visuais.

0 que os historiadores da arquitetura identificaram não é, portanto, o simples reconhecimento de que a leitura de outras fontes permitiria revelar autores ou arquiteturas subalternas, assim como temas ou problemas apagados por uma narrativa dominante. É certo que a Nova História (Novais e Silva, 2013), com suas metodologias e debates críticos, foi decisiva também para a arquitetura, permitindo ampla e profunda renovação do campo, como se pode identificar nos recentes debates dos historiadores da arquitetura no Brasil (Castro e Carvalho e Silva, 2016; Guerra, 2010; Lira, 2011). 0 que se configurou, no entanto, foi o reconhecimento de que o campo ampliou seus domínios para além do objeto construído, o tectônico, a materialidade, que tradicionalmente ocupava o centro da reflexão. 0 entendimento do que vem a ser arquitetura sofreu, assim, uma ampliação, já que manifestações visuais e textuais dedicadas à disciplina passaram a ocupar um lugar importante na configuração do campo. Essa dimensão é muito bem delineada por Beatriz Colomina: 
A arquitetura, diferentemente da construção, é um ato crítico e interpretativo. Tem uma condição linguística diferente da prática, da construção. Um edifício é interpretado quando seus mecanismos e princípios retóricos são revelados. Essa análise pode ser realizada de várias maneiras diferentes, de acordo com as formas dos diferentes tipos de discurso; entre elas estão teoria, crítica, história e manifesto. Um ato de interpretação também está presente nos diferentes modos de discurso representacional: desenho, modelagem e assim por diante. A interpretação é parte integrante do ato de projetar ${ }^{4}$. (Colomina, 1988: 6).

A manifestação de Beatriz Colomina deixa clara a distinção que os historiadores da arquitetura passaram a estabelecer. Se por um lado a construção (building) permanece como dimensão prática inerente ao campo, a arquitetura (architecture) ganhou sentido enquanto manifestação retórica, articulada pelos modos de discurso. É essa também a ponderação de Jean-Louis Cohen, historiador da arquitetura, manifestada, não por menos, em entrevista à revista October, importante veículo acadêmico para a afirmação da cultura visual ${ }^{5}$. Em debate sobre o papel das exposições de arquitetura, Cohen destaca:

Em francês, existem dois termos traduzidos como "trabalho". Um é ouvrage, referente ao trabalho construído; e outro é oeuvre, referindo-se ao projeto, ao design, ao trabalho intelectual. [...] Portanto, relacionar a documentação do projeto/oeuvre com o construído/ouvrage na parede ou em algum outro lugar é um dos desafios das exposições de arquitetura. (Bois et al., 1999: 6).

As considerações de Beatriz Colomina e Jean-Louis Cohen são fundamentais para o entendimento da transformação que se operou nesse campo disciplinar, ao menos nas últimas cinco décadas. A ampliação da arquitetura em direção às práticas retóricas intelectuais aparece aqui bem delineada, tornando compreensível o interesse de tantos historiadores da arquitetura pela visualidade. Desse modo é possível entender uma série de práticas que parecem estrangeiras ao campo - especialmente para o contexto brasileiro. As práticas contemporâneas da arquitetura ganharam significativa relevância entre historiadores, críticos e os próprios arquitetos, já que são constitutivas das formas de discurso compactuadas no campo disciplinar. É desse modo que se compreende a referência de Jean-Louis Cohen às exposições, pois é também esse um dos mais importantes domínios de atuação dos arquitetos, críticos e historiadores na contemporaneidade, em que se pode produzir arquitetura em seu sentido ampliado, intelectual, no manejo de documentos essencialmente visuais.

A compreensão de arquitetura manifestada aqui por intermédio desses importantes nomes da contemporaneidade não está descolada do que se organizou nas ciências humanas. Se antes havia a preocupação de se revisitar a História com foco em novos atores e eventos até então apagados por estruturas de poder ${ }^{6}$, identificou-se que esse objetivo só seria possível 
com a incorporação de documentos de outras naturezas - como desenhos, cartas, mapas, fotografias, filmes, objetos ritualísticos e tantos outros. Com o desenvolvimento dos debates delineados pela chamada virada linguística (Rorty, 1992), logo se reconheceu que a simples perscrutação e confrontação de diversas linguagens não seria suficiente para uma leitura comprometida com a complexidade do fazer histórico. Ao contrário, as linguagens passaram a ser mobilizadas como evidências de dinâmicas culturais de certos contextos, como explica Doris Bachmann-Medick (2016: 22). Ou seja: se uma dinâmica cultural pressupõe diálogos com valores e dinâmicas específicas a um contexto, é imprescindível que se reconheçam os agenciamentos das e entre linguagens que permitam a construção de práticas e discursos. $A$ consequência dessa compreensão não foi apenas o reconhecimento da especificidade de cada uma das linguagens, mas também dos agenciamentos suscitados por elas. Afinal, o acesso que temos a um dado evento ou manifestação cultural — uma construção, por exemplo, para não perder de vista o debate da arquitetura — é mediado por representações, que possuem valores e significados compactuados culturalmente, bem como mobilizados de maneiras específicas por determinados grupos.

O novo conjunto de suportes documentais colocou em evidência, portanto, não apenas aquilo que os documentos carregam enquanto discurso inscrito - seja o texto de uma carta ou uma imagem formada pelos sais de prata de um papel emulsionado — , mas também os circuitos de produção, circulação, controle e consumo, os modos culturais de visibilidade e invisibilidade, além das distintas maneiras de olhar. Pressupõe-se, portanto, aquilo que o arqueólogo Ulpiano Bezerra de Meneses apresentou em seu artigo "Fontes visuais, cultura visual, história visual": uma contundente leitura crítica daquilo que alguns dos mais significativos autores dedicados à cultura visual escreveram, principalmente nas últimas décadas do século XX, como Michael Baxandall, W. J. T. Mitchell e Martin Jay (Meneses, 2003). Para não perder de vista os debates suscitados pela cultura visual, o renovado panorama intelectual das ciências humanas trouxe à tona, portanto, aquilo que W. J. T. Mitchell chamou de vitalidade das imagens (Mitchell, 2005). Pensar um artefato pressupõe não apenas observar a sua dimensão material, mas também as inscrições e dimensões visuais inerentes às formas com que ele atua e formula a nossa compreensão de um dado evento, uma dada cultura. Trata-se de pensar nos objetos e nos significados a eles associados como manifestações que ocupam lugares simbólicos e práticos, já que indissociáveis de nossa vida cotidiana. É uma dimensão ritualística, que pressupõe ação para não perder de vista a influência que a antropologia assumiu no debate?

As proposições formuladas na arquitetura não se encontram mais, portanto, exclusivamente direcionadas à problematização da matéria construída ou da tectônica, mas ex- 
pandiram seus domínios. Esse reconhecimento confere nova densidade às teorias e ensaios críticos manifestados entre o fim do século XX e o início do XXI, provando que se trata de uma disciplina em transformação. Debates como os apresentados por John Rajchman (2013 [1998]), que versam sobre a importância dos diagramas como instrumentos de diagnóstico, assim como o de Antoine Picon (2013 [2004]), que destacou a influência do computador no entendimento e na própria experiência da arquitetura, representam bem a mudança e a busca por novos entendimentos associados à arquitetura. Mas parece mesmo relevante destacar a formulação de Anthony Vidler em seu ensaio "O campo ampliado da arquitetura" (2013 [2008]). Tomando como ponto de partida "A escultura no campo ampliado", ensaio de Rosalind Krauss (1979), Vidler destaca que "depois de várias décadas de autonomia autoimposta, a arquitetura ingressou há pouco em um campo bastante ampliado" (Vidler, 2008: 247), incorporando concepções de uma série de disciplinas, do paisagismo à animação digital. Nesse ponto, parece relevante ressaltar que a referência de Vidler ao trabalho de Krauss contribui com uma nova camada à formulação dessa nova epistemologia para a arquitetura. Afinal, como já citado anteriormente, Krauss, ao lado de Hal Foster, teve papel relevante na conformação dos debates da Cultura Visual, especialmente na revista October.

Mas vale destacar que esse movimento recente em direção à retórica suscitada pelas representações, expandindo o entendimento do que vem a ser arquitetura, ganhou um novo sentido ao se reconhecer que as representações ocupam lugres simbólicos e práticos, que agenciam e mobilizam a nossa compreensão sobre um determinando fato. Tal entendimento aparece também manifestado na fala de David Peyceré, conservador-chefe da Cité de l'Architecture et du Patrimoine em Paris, ao debater as práticas investigativas dos historiadores da arquitetura:

Eles [os historiadores da arquitetura] não estão usando os documentos para estudar um evento histórico ou um artefato, que levou à criação do documento; ao contrário, eles estão focados nas condições que levaram à criação do próprio documento. Os pesquisadores estão se tornando inventores, organizadores ou arquivistas. (Peyceré, 2008: 13).

Se os pesquisadores deixaram de se interessar pelos documentos como forma de estudar um evento histórico ou um artefato, passando a concentrar seus esforços em reconhecer suas condições de criação, pode-se identificar uma vez mais que o interesse não está exclusivamente na matéria ou na construção em si, mas nos agenciamentos associados aos documentos. Mas o que David Peyceré apresenta nessa passagem é ainda mais contundente para o entendimento do que se vem configurando. Entendendo-se que aquilo que é construído não pode ser lido como manifestação apartada de suas representações, já que estas são constitutivas de uma dada cultura, amplia-se a importância ocupada pelo pesquisador como aquele 
capaz de atuar como "inventor, organizador ou arquivista" de uma dada cultura arquitetônica. Nesse ponto, encontramos um paralelo importante com a História Cultural, fortemente influenciada pelos debates delineados na antropologia a partir dos anos 1980. Na clássica obra A escrita da cultura, por exemplo, o antropólogo James Clifford deixa claro que, a partir do momento em que o etnógrafo passou a ser considerado como um "escriba e arquivista", novas questões puderam ser formuladas a todas as etnografias. Ou seja: o foco analítico, na antropologia, deslocou-se do mundo representado para as "instâncias específicas de discurso" - manifestadas em perguntas fundamentais, como "Quem fala? Quem escreve? Quando e onde? Com quem ou para quem? Sob quais limites institucionais ou históricos?" (Clifford, 2016: 45). As perguntas colocadas por Clifford sinalizam, assim, para os desdobramentos que ocorreram nas ciências humanas e que mobilizaram também os debates dos arquitetos.

Se os aqui já citados trabalhos de Beatriz Colomina e Jean Louis-Cohen manifestam um desenvolvimento em favor dos debates linguísticos, mas também os seus agenciamentos numa dada cultura, não faltam exemplos de como os historiadores da arquitetura se valeram de discussões das ciências humanas para reposicionar o próprio campo. Gorge Baird (1969), por exemplo, aborda a importância da antropologia para um entendimento da arquitetura como evidência em um texto social, além de mobilizar os ensaios críticos de Roland Barthes ${ }^{8}$ sobre a linguagem, como fizeram Diana Agrest (1974), Jorge Silvetti (1998 [1977]) e Alan Colquhoun (1998 [1978]). Barthes é também estruturante para as proposições críticas de Manfredo Tafuri — um dos mais influentes pensadores da arquitetura na segunda metade do século XX — que tem na obra de Michel Foucault um fundamento indiscutível (Tafuri, 1974). Nesse caso, é relevante destacar que obras seminais desse filósofo francês — especialmente com As palavras e as coisas (Foucault, 2007) e Arqueologia do saber (Foucault, 2009) — viriam a ser alçadas recorrentemente pelos historiadores da arquitetura. Foram esses diálogos com as ciências humanas que tornaram possível a mudança percebida por Peyceré na prática dos historiadores, incluindo debates sobre a linguagem, as instâncias de discurso, bem como as estruturas de poder. Essa mudança epistemológica permitiu a emergência de três grandes domínios de atuação e reflexão entre historiadores, críticos e arquitetos - domínios marcadamente associados à visualidade. Ainda que cada um deles tenha suas particularidades, é relevante destacar alguns de seus aspectos históricos e práticos, permitindo que se reconheça a articulação entre eles e a centralidade ocupada pela cultura visual em suas formulações.

0 primeiro desses três domínios diz respeito aos arquivos. Se a arquitetura tem na linguagem e nos agenciamentos entre discursos o elemento estruturante para a atuação intelectual e projetiva de historiadores, críticos e arquitetos, deter arquivos passou a ser moeda política no contexto contemporâneo. Afinal, como apresentado por Jacques Derrida em sua 
clássica obra Mal de arquivo, se o arquivo é o futuro da história, detê-lo, controlá-lo ou rearranjá-lo é condição imprescindível a qualquer grupo, instituição ou, até mesmo, país (Derrida, 1995). Não é coincidência, portanto, que a história da arquitetura e das instituições ligadas a essa disciplina, nas últimas décadas, tenha reconhecido os arquivos como estruturas de poder de primeira ordem. Nesse sentido, vale destacar que os desenhos de arquitetura no início dos anos 1970 assumiram um estatuto de obra de arte, o que contribuiu não apenas para a consolidação do chamado sistema de estrelas (Cohen, 2013) ${ }^{9}$ da arquitetura, mas também para a inclusão de seus desenhos no sistema das artes. 0 caso emblemático dessa transformação é a exposição Five architects: Eisenman, Graves, Gwathmey, Hejduk, Meier, organizada em 1969 pelo Museum of Modern Art (MoMA) e copilada em catálogo editado por Arthur Drexler, então curador de arquitetura do museu (Drexler, 1972). Três anos mais tarde, Drexler realizou a curadoria, também no MoMA, da exposição The Architecture of the École des Beaux-Arts, que viria a estabelecer um novo patamar para os desenhos de arquitetura. Ao contrário do que se configurou em torno da obra dos cinco arquitetos de Nova York, essa exposição não apenas contribuiu para a emergência de um sistema de galerias especializadas em arquitetura, mas foi também um marco de virada no entendimento dos desenhos como artefatos que atuam e constroem a cultura, do mesmo modo que permitem a sua leitura (Kauffman, 2018). Ou seja: antes de revelarem aspectos pictóricos do desenho em si — como manifestação plástica autônoma - , eles passaram a ser lidos como documentos, nos quais a visualidade se apresenta como manifestação que revela o seu valor entre práticas e dinâmicas culturais, numa chave marcada pelos debates da antropologia.

Os desenhos de arquitetura passaram, assim, a ocupar um lugar distinto na cultura ocidental, no momento em que a crise do petróleo impunha uma devastadora recessão econômica, não apenas restringindo as oportunidades de construção, mas transformando o mundo do trabalho (Botelho, 2008). Esse contexto favoreceu a emergência de novos equipamentos culturais fomentados pelos Estados, como também por empresas, que buscavam alternativas de aquecimento econômico ou valorização de suas marcas. Foi esse o cenário de criação do Centro Georges Pompidou, em Paris, inaugurado em 1977, que evidenciou o lugar dos museus e instituições culturais nesse novo contexto. Mobilizados pelas proposições do departamento de arquitetura desse centro cultural, arquitetos e historiadores reconheceram ali um modelo de atuação e correram para revisitar suas práticas, criar instituições, museus e, principalmente, constituir arquivos de arquitetura. Numa clara reação ao efeito Beaubourg, dois anos mais tarde já se havia formado a International Confederation of Architecture Museums (ICAM), coordenada por dirigentes de museus, arquivos e instituições dedicadas à arquitetura, e que, desde então, vem sendo a ponta de lança na proposição de políticas de aquisição, 
conservação e preservação da arquitetura, especialmente no que se refere aos seus arquivos (Giral, 2009) ${ }^{10}$. A criação da ICAM representa uma virada importante, na medida em que foi ela a responsável por consolidar princípios de atuação e um código de ética compartilhados, contribuindo para a criação de outros organismos e instituições, como a Section of Architectural Archives (SAR), criada em 1990 na International Confederation of Archives (ICA).

A transformação imposta a partir do fim dos anos 1970 fez com que antigas instituições culturais dedicadas à arquitetura tivessem de revisitar suas práticas. Foi esse o caso do Museum of Finnish Architecture, criado em 1956, como também o do Swedish Centre for Architecture and Design, criado em 1962, que também participaram ativamente dos debates promovidos pela ICAM e que, por isso, tiveram de reposicionar suas ações institucionais. Mas as mudanças impostas ao campo contribuíram também para a criação de novas instituições privadas e públicas. É esse o contexto de criação, por exemplo, do Canadian Centre for Architecture (CCA) (Beasley, 2009) ${ }^{11}$ em Montreal, idealizado e dirigido pela arquiteta Phyllis Lambert, um dos nomes mais enérgicos na preservação de arquivos e uma das principais lideranças da ICAM. Criado também no ano de 1979, o CCA é, sem sobra de dúvidas, uma das mais importantes instituições culturais dedicadas à arquitetura, seja por ter constituído um dos mais significativos arquivos de arquitetura, seja por suas políticas de pesquisa, que 0 consolidaram como um dos mais relevantes agentes na proposição de agendas para a arquitetura, ao lado universidades como Princeton e Harvard. Outra instituição de grande projeção, surgida nesse mesmo momento, é reconhecidamente o Netherlands Architecture Institute (NAI). O NAI foi uma iniciativa do próprio governo holandês, que identificou na arquitetura um ativo econômico de grande valor político.

0 efeito NAI, como colocado por Sergio Figueiredo (2016), representa bem a importância que essa instituição assumiu não apenas para a Holanda, mas para todo um sistema cultural, que reconheceu em equipamentos dedicados à arquitetura agentes imprescindíveis à nova dinâmica político-financeira de um mundo globalizado e pautado pela criação da União Europeia, em 1992 ${ }^{12}$. Nesse contexto, deter arquivos passou a ser uma moeda político-diplomática, na medida em que permite a invenção de novas arquiteturas. A corrida aos arquivos de arquitetura e a eclosão de museus e instituições culturais representam bem o reconhecimento da visualidade como elemento-chave no agenciamento de novas práticas. No entanto, é importante destacar que esses arquivos não foram tomados como um passado cristalizado ou estabilizado. Ao contrário, reconheceu-se que tais estruturas funcionam como ativos para a proposição de novas agendas, indissociáveis de debates políticos e culturais. É nesse contexto que é possível identificar a emergência do segundo importante domínio que viria a reconfigurar a cultura arquitetônica: o das exposições de arquitetura. 
A historiografia é taxativa ao tomar as exposições de arquitetura como manifestações fundamentais na consolidação de princípios associados ao campo. Se a Grande Exposição, realizada em Londres no ano de 1851, é um marco singular da industrialização e da racionalização da arquitetura, as exposições da primeira metade do século XX são também recorrentemente apresentadas como estruturas importantes na definição de linguagens tectônicas, que expressam posições políticas de estados (Al Assal, 2014; Greenhalgh, 1988). Mas, novamente, a partir do fim dos anos 1970, as exposições de arquitetura ganharam outro significado. Em vez de se apresentarem como dispositivos de exibição daquilo que se construía ou se pretendia construir, as exposições foram tomadas como lugares de pesquisa ou proposição de novas agendas políticas e culturais. É essa a ponderação de Barry Bergdoll, professor de história da arte na Universidade de Columbia e curador de arquitetura no MoMA. Debatendo a exposição como um meio de pesquisa histórica, Bergdoll destaca que "[...] os materiais expostos são solicitados não apenas a representar edifícios ausentes, mas também a invocar contextos ou ambientes ausentes em que a arquitetura participou ou participa - histórica, urbana ou teoricamente" (Bergdoll, 1998: 257). É certo que ainda persistem exposições de arquitetura dedicadas a representar objetivamente edifícios, modelo este que está longe de ser aposentado. Mas a reflexão de Bergdoll deixa clara a mudança ocorrida, uma vez que o contexto é muitas vezes mais relevante à reflexão e que, por isso, importa pensar nos documentos como artefatos - essencialmente visuais - que povoam e são mobilizados em meio à cultura arquitetônica. $E$, nesse caso, Bergdoll reconhece a importância da relação com a cultura visual, uma vez que os "argumentos e percepções são feitos com objetos e imagens, e não prioritariamente com palavras" (Bergdoll, 1998: 257).

Esse reconhecimento das exposições como meios laboratoriais para a reflexão na arquitetura - seja ela uma leitura histórica ou uma proposição de agenda contemporânea - tem marcos de virada importantes. Para Véronique Patteeuw e Léa-Catherine Szacka, as exposições The Architecture of the École des Beaux-Arts (1975), Roma Interrotta (1978) e The Presence of the Past (1980) "desempenharam um papel fundamental na proclamação de uma nova sensibilidade na arquitetura, e isso antes que a maioria dos edifícios pós-modernos vissem a luz do dia" (Patteeuw e Szacka, 2018: 1). The Presence of the Past - a primeira Bienal de Arquitetura de Veneza — é, sem sombra de dúvidas, um marco irredutível dessas transformações. Chamados para contribuírem para o debate, os arquitetos não apresentaram apenas desenhos, maquetes ou fotografias de seus novos projetos. Ao contrário, foram mobilizados a colaborar com instalações que conformariam a importante Strada Novissima, a principal exposição apresentada na Corderie dell'Arsenale, um grande aparato expositivo que se tornaria um manifesto de grande efeito e projeção visual em prol da pós-modernidade. 
A criação da Bienal de Arquitetura de Veneza é, sem sombra de dúvidas, um marco referencial. Se por um lado ela evidencia esse processo de "bienalização" da cultura, sobre o qual atuam pesadas forças econômicas, por outro destaca o reconhecimento de que as exposições passaram a ser mobilizadas como "veículos de produção do conhecimento e debates intelectuais", como destacado por Paul O’Neill (2016: 81) e igualmente manifestado nas indagações de Giovanna Borasi:

A exposição é um objetivo per se, ou uma ferramenta estratégica entre outras para fomentar ideias, desafiar posições, introduzir novos temas, questionar tópicos atuais na tentativa de, em última instância, desenvolver teorias e mudar as práticas atuais? (Borasi, 2015: 29).

O protagonista do debate não é aquilo que é construído, mas a proposição ou o questionamento realizado pelo arquiteto, crítico ou historiador, um curador por excelência, alçado a intelectual. Nas artes, esse lugar da curadoria como espaço de ação propositiva laboratorial — projetivo, para reconhecermos aqui esse domínio associado à arquitetura — é um marco importante na exposição Les Immatériaux — sob curadoria do filósofo Jean-François Lyotard, realizada no Beaubourg em 1985 (Rajchman, 1985). Usar aparato expositivo para debater a imaterialidade foi uma forma radical de se colocar em evidência esse lugar propositivo assumido pela curadoria e pelas exposições.

$\mathrm{Na}$ arquitetura, esse aspecto foi sendo elaborado nos anos posteriores à primeira Bienal de Arquitetura de Veneza e, segundo Léa-Catherine Szacka, encontrou sua manifestação singular na exposição Herzog \& de Meuron: Archeology of the Mind, apresentada no CCA, em 2002 (Szacka, 2016: 21-22). Para essa exposição, o curador responsável, Philip Ursprung, optou por excluir completamente as representações da arquitetura. A opção da curadoria foi apresentar o processo projetivo dos arquitetos, como numa operação arqueológica, mas no interior do arquivo de arquitetura, e o público era mobilizado a reconstruir as elaborações mentais com base em fragmentos materiais preservados nos arquivos. Esse aspecto é muito relevante, pois representa o extremo oposto das exposições referenciadas naquilo que é construído e, desse modo, bem demonstra como as exposições de arquitetura se tornaram práticas arquitetônicas em seu sentido intelectual, no qual os pressupostos da cultura visual são manejados enquanto ferramentas metodológicas. E vale dizer que esse processo de elaboração mental, intelectual, organizado por meio das exposições, tem relação estreita com as formas de mobilizar os arquivos e sua documentação. Assim, arquivo e exposição parecem entrelaçados, revelando sua importância nesse novo contexto ampliado da arquitetura.

0 terceiro e último domínio que bem caracteriza essa nova cultura arquitetônica aparece como elemento que consolida discursos entre arquivos e práticas expositivas. Nesse caso, é reveladora a manifestação dos curadores da Bienal de Arquitetura de Oslo de 2016, que 
destacaram que o evento era apenas uma plataforma para a realização de uma série de ações reflexivas, tais como seminários, projetos e propostas de ensino e pesquisa. Trata-se de uma clara manifestação dessa equação prática, inventiva, manejada pelos arquitetos. Não por menos, para os curadores dessa Bienal, o catálogo da exposição — um livro por excelência — foi tratado como projeto protagonista, pelas suas possibilidades construtivas, mas também palas suas subsequentes autonomia e projeção internacional (Szacka, 2019: 72-73). Léa-Catherine Szacka (2019: 26) reconhece esse fenômeno dos livros como espaços paralelos das exposições, mas é Barry Bergdoll que identifica a autonomia dos catálogos/livros como laboratórios de proposição e experimentação intelectual, tendo "[...] evoluído dramaticamente de uma lista de verificação [...] para elaborar 'ligações'" (Bergdoll, 1998: 257).

A relação dos livros com a arquitetura é longeva e vem sendo esmiuçada pelos historiadores. Mario Carpo, por exemplo, não deixa dúvidas de que o livro foi o grande aparato de transformação na arquitetura, capaz de permitir o surgimento da cultura renascentista. Foi com a invenção de Johannes Gutenberg que o tratado de Vitrúvio pôde ser replicado, permitindo a emergência de tratados renascentistas, como os de Leon Battista Alberti e Sebastiano Serlio (Carpo, 2001). 0 debate dos livros de arquitetura foi também enfrentado pelo historiador, crítico, curador e editor português André Tavares, no livro Uma anatomia do livro de arquitetura, que também mereceu uma exposição no CCA. Tavares explicita a condição projetiva do artefato livro, ao destacar que:

As qualidades dos livros parecem ser suficientes para comunicar ideias de arquitectura que thes estão subjacentes, independentemente dos edifícios que lhes dão substância ou de aspectos como os seus diferentes tamanhos, rugosidade ou delicadeza dos papéis, a tinta negra de algumas gravuras ou a qualidade etérea das litografias. (Tavares, 2016: 13).

Se os discursos transmitidos pelos livros, enquanto texto inscrito no artefato, foram decisivos para a transformação de uma cultura construtiva, Tavares é assertivo na caracterização desses artefatos como objetos visuais, que carregam em si discursos a ser manejados numa dada cultura. 0 entendimento do artefato livro como elemento que não só age numa dada cultura, mas que, também por isso, nos permite reconhecer práticas estabelecidas em determinados períodos históricos, alçou o livro, na contemporaneidade, à condição de documento histórico, mas também de espaço projetivo - um lugar de atuação intelectual dos próprios arquitetos. Foi nessa perspectiva que o já citado trabalho de Beatriz Colomina colocou a revista L'Esprit Nouveau no centro da análise do trabalho do arquiteto Le Corbusier (Colomina, 1994). Colomina foi das historiadoras que melhor reconheceu a centralidade desse artefato, expandindo seus debates para as radicais publicações dos arquitetos pós-modernos, entre os anos 1960 e 1970 (Colomina e Buckley, 2010). Nesse caso, a radicalidade de suas propostas 
construtivas não se apresenta apenas latente na materialidade possível de suas construções idealizadas, mas na própria materialidade dos livros. Novas composições, o uso de processos gráficos pouco usuais, tintas, formatos e tipos de papéis são apenas alguns dos aspectos radicais dessa nova cultura arquitetônica, expressa também em suas "construções" impressas, como destaca André Tavares: "Ao longo desta história, e para além das particularidades do mercado editorial [...], os arquitetos parecem ter sabido fazer livros como se estes fossem construções" (Tavares, 2016: 388).

As pesquisas contemporâneas sobre os livros e impressos enquanto artefatos visuais representam uma vez mais essa transformação epistemológica ocorrida na arquitetura. Mas é necessário identificar, também, que esse movimento dos historiadores e críticos é fruto do crescente entendimento, por parte dos arquitetos, de que o livro passou a ser também um espaço de pesquisa, projeto, uma arquitetura. Tal reconhecimento tem expressão eloquente no livro S.M.L.XL., elaborado pelo arquiteto holandês Rem Koolhaas em parceria com 0 designer Bruce Mau (1998). Aqui, é importante destacar que Koolhaas talvez seja o maior representante dessa geração, tendo participado da primeira Bienal de Arquitetura de Veneza e sido alçado a protagonista pela agenda cultural organizada pelo governo holandês nos anos 1990. O livro de Koolhaas não é, portanto, excêntrico ao contexto que se configurou, mas uma reiteração do fato de que a arquitetura passou a operar por meio de um conjunto de outras mídias enquanto espaços projetivos, intelectuais, como destacado por Gabriel Girnos de Souza (2015). Esse entendimento foi incorporado à prática de outros tantos escritórios de arquitetura, fazendo com que não tenha sido apenas o Office for Metropolitan Architecture (OMA) a dedicar anos de seus trabalhos à elaboração dessa e de outras obras impressas, mas toda uma geração de arquitetos. Tal contexto leva ainda ao reconhecimento de um fenômeno contemporâneo, a emergência de editoras ou selos editoriais dedicados à arquitetura. Dos museus e centros culturais voltados a essa área - como o CCA e o NAI, que estabeleceu o selo nai010 publishers — às universidades - como o MIT e a Columbia University, com seu selo Columbia Books on Architecture and the City — e editoras especializadas - como a Lars Müller Publishers e a Editora Dafne — , todos passaram a investir grande atenção e recursos às publicações dos arquitetos, enfrentando suas particularidades e especificidades. Afinal, construir e publicar livros passou a ser condição inerente à atuação dos arquitetos nesse contexto expandido da arquitetura.

0 reconhecimento dessa natureza contemporânea da arquitetura é fruto de uma série de mudanças epistemológicas do campo, fortemente influenciado por novos problemas das ciências humanas, assim como por forças político-econômicas que ocuparam a agenda cultural global, expressas com mais eloquência no eixo Europa- América do Norte. Assim, 
a arquitetura vem-se organizando entre esses três domínios: os arquivos, as exposições e os livros impressos. Aqui, evidencia-se esse lugar desempenhado pela cultura visual, na qual os debates não se ocupam simplesmente de imagens, mas de objetos manejados em culturas. Nesse sentido, vale retomar a consideração de James Clifford, cruzada pela avaliação de David Peyceré. Se ao antropólogo ou ao historiador da arquitetura contemporânea é requisitado o lugar de escriba ou arquivista, compreender tais formas de expressão e configuração dos discursos é pressuposto básico para o ato reflexivo, projetivo. Trata-se do fundamento contemporâneo das formas de ação de arquitetos, críticos e historiadores, que foram alçados a inventores, organizadores e arquivistas. Arquitetos projetistas não mais se dedicam apenas à prática construtiva ligada aos edifícios, mas trabalham de forma imbricada com a visualidade.

0 entendimento dessa transformação da arquitetura reconsidera ou reposiciona o lugar ocupado pelo historiador. Tendo a atuação do arquiteto - assim como a do crítico e do historiador - se deslocado em direção a esses três domínios, a natureza do trabalho historiográfico precisou também reavaliar seus pressupostos, já que agora se associa a esse entendimento ampliado da arquitetura como prática intelectual. Desse modo, algumas mudanças impuseram-se e permanecem pressionando o campo em direção a novas práticas e reorientações, entre as quais parece importante destacar as seguintes: primeiramente, a mudança de foco investigativo sobre novos objetos pressupõe a avaliação de condições culturais e materiais potencialmente novas e específicas a cada um deles; como consequência, é inevitável que outras formas de pesquisa sejam postas em prática - que pressupõem atuação em arquivos, exposições e publicações - , reconhecendo que se trata de manifestações potentes dos problemas contemporâneos. Por fim, a perspectiva laboratorial presente na dinâmica de pesquisa torna-se, inevitavelmente, imprescindível ao ensino, não só para a história, mas para o campo como um todo. Não foi por menos que a cultura visual se estabeleceu como lugar singular, já que não só é fundamental para a leitura do que se vem manifestando, mas também permite a utilização de suas metodologias nos enfrentamentos contemporâneos. É por meio dela, afinal, que se podem pensar, elaborar e propor novas questões para o mundo.

Conflitos de interesse: nada a declarar.

Fonte de financiamento: Fundação de Amparo à Pesquisa do Estado de São Paulo, Auxílio à Pesquisa Jovem Pesquisador. 


\section{NOTAS}

10 debate sobre as definições e metodologias da cultura visual é extenso e não caberia no escopo deste artigo. De todo modo, destaco importantes trabalhos para uma introdução qualificada: o livro Cultura visual \& história (Schiavinatto e Costa, 2016) e o artigo "A virada e a imagem: história teórica do pictorial/iconic/visual turn e suas implicações para as humanidades" (Santiago Júnior, 2019).

2 Disponivel em: <https://www.youtube.com/watch?v=0pDE4VX_9Kk>. Acesso em: 28 ago. 2020.

3 Os três princípios centrais da boa arquitetura clássica, segundo o tratado de Vitrúvio, seriam: firmitas (solidez), utilitas (funcionalidade) e venustas (beleza) (Polião, 2009).

4 Tradução livre de: "Architecture, as distinct from building, is an interpretative, critical act. It has a linguistic condition different from the practical one of building. A building is interpreted when its rhetorical mechanism and principles are revealed. This analysis may be performed in a number of different ways, according to the forms of different types of discourse; among these are theory, criticism, history and manifesto. An act of interpretation is also present in the different modes of representational discourse: drawing, model making and so on. Interpretation is alto integral to the act of projecting".

5 Rosalind Krauss, uma das entrevistadoras de Cohen, e Hal Foster foram os editores responsáveis pela edição do volume 77, do verão de 1996, reconhecido como edição estruturante para a organização dos debates em torno da Cultura Visual (Schiavinatto e Costa, 2016).

6 Numa marcada relação com a perspectiva apresentada por Michael Foucault, como destacado por Lynn Hunt (Foucault, 2009; Hunt, 1995).

7 Importante destacar, nesse caso, os trabalhos de Natalie Zemon Davis, na articulação entre antropologia e história, especialmente seu livro 0 retorno de Martin Guerre (Davis, 1984).

8 Baird considera os trabalhos de Barthes como a melhor introdução ao pensamento estruturalista de Claude Lévi-Strauss.

9 Ver capítulo "A temporada pós-moderna".

10 Para mais detalhes sobre a ICAM, ver: <https://wwww.icam-web.org>. Acesso em: 28 ago. 2020.

11 Para mais detalhes sobre o CCA, ver: <https://www.cca.qc.ca/en>. Acesso em: 28 ago. 2020.

12 Vale também aqui uma consideração sobre o chamado "efeito Bilbao", provocado pela construção do museu Guggenheim, projetado por Frank Gehry, no País Basco. Nesse caso, trata-se da espetacularização das cidades, com a construção de atrativos equipamentos culturais (Jameson, 1992). 


\section{REFERÊNCIAS BIBLIOGRÁFICAS}

AGREST, D. Design versus non-design [1974]. In: HAYS, M. (ed.). Architecture theory since 1968. Cambridge: The MIT Press, 1998. p. 198-214.

AL ASSAL, M. R. B. Arenas nem tão pacíficas: arquitetura e projetos políticos em exposições universais de finais da década de 1930. 2014. Tese (Doutorado em Arquitetura e Urbanismo) — Faculdade de Arquitetura e Urbanismo da Universidade de São Paulo, Universidade de São Paulo, São Paulo, 2014.

BACHMANN-MEDICK, D. Cultural turns: new orientations in the study of culture. Berlin, Boston: Walter de Gruyter GmbH, 2016.

BAIRD, G. "La dimension Amoureuse" in architecture [1969]. In: HAYS, M. (ed.). Architecture theory since 1968. Cambridge: The MIT Press, 1998. p. 40-55.

BEASLEY, G. Interview with Phyllis Lambert. Icam Print, Netherlands, v. 3, p. 34-39, 2009.

BERGDOLL, B. Curating architecture. The Journal of the Society of Architectural Historians, [S.I.], v. 57, n. 3, p. 257-366, 1998. https://doi.org/10.2307/991345.

BOIS, Y.-A.; HOLLIER, D.; KRAUSS, R.; COHEN, J.-L. A conversation with Jean-Louis Cohen. October, Cambridge, v. 89, p. 3-18, 1999. https://doi.org/10.2307/779136.

BORASI, G. For architecture. In: GIGLIOTTI, R. (ed.). Displayed spaces: new means of architecture representation through exhibitions. Leipzing: Spector Books, 2015. p. 21-35.

BOTELHO, A. Do fordismo à produção flexível: o espaço da indústria num contexto de mudanças das estratégias de acumulação do capital. São Paulo: Annablume, 2008.

BOWMAN, R. Words and images: a persistent paradox. Art Journal, New York, v. 45, n. 4, p. 335-343, 1985. https://doi.org/10.2307/776809.

CARPO, M. Architecture in the age of printing: orality, writing, typography, and printed images in the history of architectural theory. Massachusetts: The MIT Press, 2001.

CASTRO, A. C. V.; CARVALHO e SILVA, J. M. DOSSIÊ - Fazer história: o estatuto das fontes e o lugar dos acervos nas pesquisas de história de arquitetura e da cidade no Brasil. Anais do Museu Paulista: História e Cultura Material, São Paulo, v. 24, n. 3, p. 11-18, set./dez. 2016. https://doi.org/10.1590/1982-02672016v24n03do.

CLIFFORD, J. Introdução: verdades parciais. In: CLIFFORD, J.; MARCUS, G. E. (orgs.). A escrita da cultura: poética e política da etnografia. Rio de Janeiro: EdUERJ, 2016. p. 31-61.

COHEN, J. O futuro da arquitetura desde 1889: uma história mundial. São Paulo: Cosac Naify, 2013.

COLOMINA, B. Introduction: on architecture, production and reproduction. In: COLOMINA, B. Architectureproduction. Series Revisions: Papers on Architectural Theory and Criticism. New York: Princeton Architectural Press, 1988. p. 6-23.

COLOMINA, B. Privacy and publicity: Modern Architecture as Mass Media. Cambridge: The MIT Press, 1994.

COLOMINA, B.; BUCKLEY, C. (eds.). Clip, stamp, fold: the radical architecture of little magazines 196X to 197X. Princeton: M+M Books, Media and Modernity Program, Princeton University, 2010. 
COLQUHOUN, A. From bricolage to myth, or how to put Humpty-Dumpty together again [1978]. In: HAYS, M. (ed.). Architecture theory since 1968. Cambridge: The MIT Press, 1998. p. 332-347.

DAVIS, N. Z. The return of Martin Guerre. Massachusetts: Harvard University Press, 1984.

DERRIDA, J. Archive fever: a Freudian impression. Chicago: The University of Chicago Press, 1995.

DESÍGNIO: REVISTA DE HISTÓRIA da Arquitetura e do Urbanismo, São Paulo, n. 11/12, mar. 2011. Dossiê: História, Historiografias, Historiadores.

DREXLER, A. et al. Five architects: Eisenman, Graves, Gwathmey, Hejduk, Meier. Nova York: Oxford University Press, 1972.

FIGUEIREDO, S. M. The NAi Effect: creating architecture culture. Rotterdam: nai010 publishers, 2016.

FOUCAULT, M. This is not a pipe. Berkeley: University of California Press, 1983.

FOUCAULT, M. As palavras e as coisas: uma arqueologia das ciências humanas. São Paulo: Martins Fontes, 2007.

FOUCAULT, M. A arqueologia do saber. Rio de Janeiro: Forense Universitária, 2009.

GIRAL, A. International Confederation of Architectural Museums, a History. Icam Print, Netherlands, v. 3, p. 8-15, 2009.

GREENHALGH, P. Ephemeral Vistas: The Expositions Universelles, Great Exhibitions and World's Fairs, 18511939. Manchester: Manchester University Press, 1988.

GUERRA, A. (org.). Textos fundamentais sobre história da arquitetura moderna brasileira - Parte 1. São Paulo: Romano Guerra Editora, 2010. (Coleção Bolso RG, 1 e n. 2).

HUNT, L. (ed.). A nova história cultural. Martins Fontes: São Paulo, 1995.

JAMESON, F. Postmodernism, or, the cultural logic of late capitalism. Durham: Duke University Press, 1992.

KAUFFMAN, J. Drawing on architecture: the object of lines, 1970-1990. Cambridge: The MIT Press, 2018.

KRAUSS, R. Sculpture in the expanded field. October, Cambridge, n. 8, p. 41, 1979.

KOOLHAAS, R.; MAU, B. S.M.L.XL. New York: Monacceli Press, 1998.

MENESES, U. T. B. Fontes visuais, cultura visual, história visual. Balanço provisório, propostas cautelares. Revista Brasileira de História, São Paulo, v. 23, n. 45, p. 11-36, 2003. https://doi.org/10.1590/S010201882003000100002.

MITCHELL, W. T. J. What do pictures want? The lives and loves of images. Chicago: The University of Chicago Press, 2005.

NOVAIS, F. A.; SILVA, R. F. Nova história em perspectiva. São Paulo: Cosac \& Naify, 2013. v. 1 e 2.

O'NEILL, P. The culture of curating and the curating of the culture(s). Massachusetts: The MIT Press, 2016.

PATTEEUW, V.; SZACKA, L. Postmodern architecture and the media: an introduction. In: PATTEEUW, V.; SZACKA, L. (eds.). Mediated messages: periodicals, exhibitions and the shaping of postmodern architecture. London: Bloomsbury, 2018. p. 1-21. 
PEYCERÉ, D. La cartographie d'un monde nouveau. In: PEYCERÉ, D.; WIERRE, F. (eds.). Architecture et archives numériques: I'architecture à l'ère numérique, un enjeu de mémoire. Paris: Éditions Infolio, 2008. p. 13.

PICON, A. A Arquitetura e o virtual: rumo a uma nova materialidade [2004]. In: SYKERS, A. K. (org.). 0 campo ampliado da arquitetura. São Paulo: Cosac Naify, 2013. p. 206-220.

POLIÃO, M. V. Vitrúvio: Tratado de Arquitectura. Lisboa: IST Press, 2009.

RAJCHMAN, J. The Postmodern Museum. Art in America, v. 73, n. 10, p. 110-117, Oct 1985.

RAJCHMAN, J. Um novo pragmatismo? [1998]. In: SYKERS, A. K. (org.). O campo ampliado da arquitetura. São Paulo: Cosac Naify, 2013. p. 74-83.

RATTENBURY, K. This is not architecture. London: Routledge, 2002.

RORTY, R. M. (ed.). The linguistic turn: essays in philosophical method. With Two Retrospective Essays [1967]. Chicago: University of Chicago Press, 1992.

SANTIAGO JÚNIOR, F. C. F. A virada e a imagem: história teórica do pictorial/iconic/visual turn e suas implicações para as humanidades. Anais do Museu Paulista: História e Cultura Material, São Paulo, v. 27, e08, 2019. https://doi.org/10.1590/1982-02672019v27e08.

SCHIAVINATTO, I. L. F; COSTA, E. A. Cultura visual: apontamentos sobre um campo disciplinar. In: SCHIAVINATTO, I. L. F.; COSTA, E. A. (orgs.). Cultura visual \& história. São Paulo: Alameda, 2016. p. 9-31.

SILVETTI, J. The beauty of shadows [1977]. In: HAYS, M. (ed.). Architecture theory since 1968. Cambridge: The MIT Press, 1998. p. 262-283.

SOUZA, G. G. E. Fiç̧ões projetuais: Projeto Gráfico e Discurso Profissional em Livros Contemporâneos de Escritórios Internacionais de Arquitetura e Urbanismo. 2015. Tese (Doutorado em Design) — Departamento de Artes \& Design, Pontifícia Universidade Católica do Rio de Janeiro, Rio de Janeiro, 2015.

SZACKA, L. Exhibiting the postmodern: the 1980 Venice Architecture Biennale. Venezia: Marsilio Editori, 2016.

SZACKA, L. Biennials/triennials: conversations on the geography of itinerant display. New York: Columbia Books on Architecture and the City, 2019.

TAFURI, M. L'Architecture dans le Boudoir: the language of criticism and the criticism of language. Oppositions, New York, v. 3, 1974. p. 37-62.

TAVARES, A. Uma anatomia do livro de arquitectura. Porto: Dafne, 2016.

VENTURI, R.; SCOTT BROWN, D.; IZENOUR, S. Learning from Las Vegas. Massachusetts: The MIT Press, 1972. VIDLER, A. O campo ampliado da arquitetura [2008]. In: SYKERS, A. K. (org.). O campo ampliado da arquitetura. São Paulo: Cosac Naify, 2013. p. 243-251. 